\section{PTH-87 CURRENT TREATMENT MODALITIES AND MEDIAN SURVIVAL FOR PATIENTS WITH GASTRIC CANCER AND ISOLATED PERITONEAL METASTASIS}

\begin{abstract}
${ }^{1,2}$ Adler Shing Chak Ma*, ${ }^{1,3}$ Stephen Lam, ${ }^{1}$ Eman Otify, ${ }^{1}$ Abdulfatah Yousfi, ${ }^{3}$ Adam Stearns, ${ }^{3}$ Bhaskar Kumar. 'Norwich Medical School, University Of East Anglia, Norwich, UK; ${ }^{2}$ Department of Gastroenterology, James Paget University Hospital NHS Foundation Trust, Great Yarmouth, UK; ${ }^{3}$ Department of Surgery, Norfolk and Norwich University Hospital NHS Foundation Trust, Norwich, UK
\end{abstract}

10.1136/gutjpl-2021-BSG.266

Introduction Gastric cancer with peritoneal metastases carries a median survival of only 3-7 months without treatment, and no treatment modality in standard practice appears to improve survival beyond a few months. In order to measure the efficacy of emerging modes of treatment, it is essential to describe the treatments patients currently receive and the impact of these on survival - data for which is poorly described in the literature and lacking in the UK setting.

Methods This was a single hospital-based retrospective cohort study which included 50 patients who received a diagnosis of gastric adenocarcinoma with isolated peritoneal disease between the dates 21 March 2012 to 14 January 2020 at a tertiary referral centre. We calculated median survival time for all patients and also by treatment modality.

Results The mean age of patients was 71 years (range 44-90 years). Overall, 26 patients (52\%) received systemic chemotherapy, three $(6 \%)$ gastrectomy, six $(12 \%)$ endoscopic stenting and $15(30 \%)$ best supportive care. Overall median survival was 6.6 months (IQR 2.4-19.3). At a mean of 16.4 months follow-up, 43 patients (86\%) had died. Median survival by treatment modality was as follows: supportive care $(2.4$ months, IQR 1.2-5.1), endoscopic stent (5.7 months, IQR 3.671.2), systemic chemotherapy (11.2 months, IQR 3.7-21.5), and palliative gastrectomy (15.1 months, IQR 6.6 to 55.9 ).

Conclusions Our results confirm the poor prognosis of gastric cancer patients with isolated peritoneal disease with available treatments only extending survival by a maximum of 4 to 8 months, highlighting the desperate need for new treatment modalities.

\section{PTH-88 HELICOBACTER PYLORI ERADICATION RATES IN A UK LOCAL POPULATION: TIME FOR A STRATEGY CHANGE?}

Olivia Greenham*, Neel Kapoor, Jana Waloszkova, Diza Gonclaves, Mohamed Shariff, Anthony Leahy. West Hertfordshire Hospitals Trust, Watford, UK

\subsection{6/gutjnl-2021-BSG.267}

Introduction Helicobacter pylori (HP) is a treatable human pathogen with a high prevalence throughout the world. Infection causes peptic ulcer disease, lymphoma and gastric carcinoma, hence the importance of eradication. Antibiotic resistance and human migration present ongoing therapeutic challenges despite decreasing incidence in the UK. We assessed our local HP eradication rates to identify if we need to alter our local treatment strategy.

Methods We performed a retrospective analysis of patients who had a urea breath test (UBT) between March 2019 to January 2021. Patients completed 1st line triple therapy (FLTT) after a positive HP result and had a UBT 6-8 weeks later to assess HP eradication. If patients tested positive they completed further treatments of 2 nd line triple therapy (SLTT) and 3rd line quadruple therapy (TLQT). Each patient had a post treatment UBT performed 6-8 weeks after completion of each therapy and if positive, progressed to the next treatment. Patients who remained positive despite TLQT, had a gastroscopy and biopsies taken for microscopy, culture and sensitivity (MC\&S).

Results 151 patients had UBTs following FLTT. 111 (74\%) had successful eradication with either FLTT or a combination of FLTT and SLTT. 40 (26\%) received quadruple therapy. Out of these, 24 had UBTs performed following treatment. 11 (46\%) had a positive UBT after quadruple therapy. 19 patients had MC\&S performed. HP was identified in 11 samples with $5(45 \%)$ showing resistance to clarithromycin and 10 (91\%) to metronidazole.

Conclusions Our data demonstrates that we need to improve our ability to eradicate HP. Up to a quarter of patients failed FLTT and SLTT. There were also high rates of treatment failure among patients who received TLQT. Contributing factors may include self-selection and underlying microbial resistance.

The samples sent for MC\&S showed a high rate of resistance to metronidazole. A UK study in 2010 Savoldi et al. looked at antimicrobial resistance in $\mathrm{HP}$ and found resistance to metronidazole as high as $88 \%$. This again suggests that microbial resistance to current treatment regimens is a concern for HP eradication. MC\&S is an accurate method for predicting treatment success. NICE guidelines advise that a biopsy is only indicated in certain circumstances including a known allergy, high local resistance rates, and those who remain $\mathrm{HP}$ positive despite 2 courses of antibiotics. There are also additional associated costs. However, local resistance rates are largely unknown and using MC\&S may negate the need for multiple rounds of testing, antibiotics and help to reduce $\mathrm{HP}$ antibiotic resistance.

We have implemented new guidelines at our Trust to use 1st line quadruple therapy with eradication failures offered MC\&S. We plan to reaudit this treatment strategy change at a later date.

\section{PTH-89 AN AUDIT OF AGIB ENDOSCOPY SERVICES IN NORTH HAMPSHIRE HOSPITALS TRUST DURING THE COVID19 PANDEMIC}

Rebecca Smith*, Ryoon Khang, Corrine Brooks, John Ramage. North Hampshire Hospitals Trust, Basingstoke, UK

\subsection{6/gutjnl-2021-BSG.268}

Introduction All healthcare services have been disrupted as a result of the COVID19 pandemic. However, the full impact of the pandemic is as yet unknown. There is little in the literature to suggest how acute gastrointestinal bleeding (AGIB) services have been impacted, despite their reliance on timely aerosol generating procedures.

Method The North Hampshire Hospitals Trust's endoscopy database was searched for cases between 01/01/2020 and 01/ 03/2021, including all emergency and urgent procedures, conducted for indications that could be related to AGIB.

805 endoscopies were identified with these search terms, however, 596 were excluded as not being related to AGIB, 1 case was excluded where there was insufficient information. 209 endoscopies were included.

Results 209 endoscopies were conducted for AGIB on 176 patients, with a mean age of $67.4($ SD16.5). The median timeto-endoscopy was 20 hours (IQR 8-39). 63.2\% of patients 
had endoscopy within 24 hours. The median time-to-endoscopy for the sub group of COVID positive patients $(n=6)$ was 17.5 hours (IQR16). A chi-squared test was used to compare the likelihood of those with COVID undergoing endoscopy $<24$ hours, compared to those who were COVID negative/unknown, and there was no significant difference (1.080 $\mathrm{p}=0.299)$.

38 re-bleeds occurred, and 37 deaths. COVID19 positivity was associated with a significantly increased risk of death when reviewed with a Chi squared test $(4.46 \mathrm{p}=0.035)$. However, COVID19 positivity was not associated with any increased risk of re-bleeding $(0.009 \mathrm{p}=0.922)$.

The number of cases of AGIB, and monthly median time to endoscopy, varied considerably but there was a strong correlation between the two over the 14 month study period, see figure 1. The month of April 2020 had the lowest number of cases at only 6 , a figure that is an outlier at $>1.5$ standard deviations from the mean for the study period. Reductions in the incidence of AGIB seemed to coincide with national lock downs.

Conclusion Patients with COVID19 and AGIB had a high risk of death, even though the data would suggest this death was not from re-bleeding. It is more likely that AGIB is a sign of physiological strain from COVID 19 infection.

We showed that AGIB cases reduced markedly at the first lockdown, which suggests that patients were not willing (or perhaps able) to present to secondary care services. This pattern was replicated during lockdowns 2 and 3. However, further review over a broader time period would be required, to further explore this observed phenomenon.

If we are using the 'time-to-endoscopy' as a surrogate marker of standards of endoscopy services, our trust appears to have offered its best AGIB service during this first lock down. This may represent reduced strain on the service. We were able to offer a comparable time-to-endoscopy to those patients who were COVID positive. The overall median time to endoscopy was comparable to the figure of 21.2 hours, found by Sian et al.(Siau et al., 2019) in the multi-site audit of 2017.

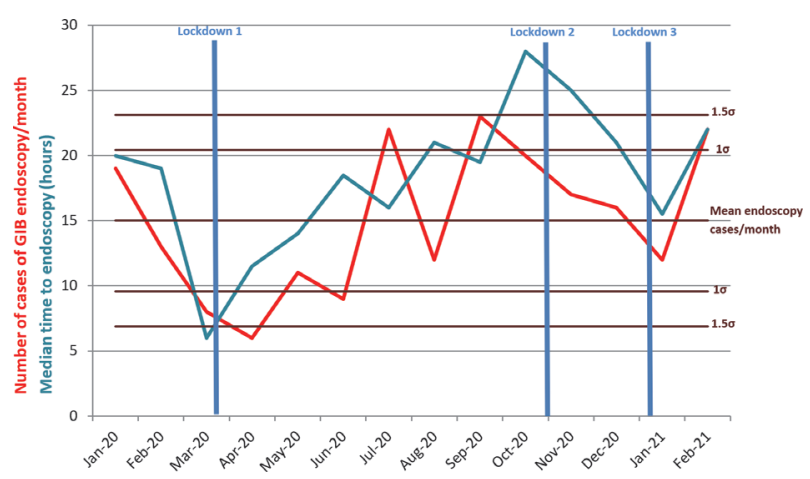

Abstract PTH-89 Figure 1

Learning from how our services have performed in this pandemic will help us prepare ourselves in case of future similar challenges.

\section{REFERENCE}

1. Siau, K. et al. (2019) 'Time to endoscopy for acute upper gastrointestinal bleeding: Results from a prospective multicentre trainee-led audit', United European Gastroenterology Journal. doi: 10.1177/2050640618811491.

\section{Pancreas and neuroendocrine}

\section{PTU-67 DIFFERENTIAL EXPRESSION OF THE POTASSIUM OF VOLTAGE-GATED CHANNEL SUBFAMILY MEMBERS, KCHN}

Xuefei Dong*. Cardiff University, Cardiff, UK

\subsection{6/gutjnl-2021-BSG.269}

Introduction Human pancreatic cancer is an intractable malignancy and is the seventh leading cause of cancer deaths worldwide and the fifth leading cause in the UK. Pancreatic cancer has ranked the 11th most common cancer in the world and the UK. The incidence and mortality of pancreatic cancer are linked with aging and is more favorable in men than in women. The dominant risk factors of pancreatic cancer remain vague and the 5 -year survival rate remains poor ( 9\%) despite clinical advances. Voltage-gated potassium (Kv) channels are known to modulate vaired functions of epitehial cells in pancreas and its ion transportation, a function partly regulated by calmodulin related proteins. The dysregulation or alterexpression of potassium channels in pancreatic cancer has yet been fully characterised. In this study, we evaluated the transcript expression levels of $\mathrm{KCNH} 1, \mathrm{KCNH} 2$ and $\mathrm{KCNH} 3$ in pancreatic cancer and their clinical and prognostic significance.

Methods Pancreatic tissues including tumour tissue sections and their adjacent normal tissue sections from a cohort of patients in our lab $(n=223)$. The gene transcripts of the three $\mathrm{KCNH}$ members were quantified in the tissues and analyses were carried out against the clinical, pathological and outcome factors of the patients.

Results We find that there is a significant difference in the expression of $\mathrm{KCNH} 1$ and $\mathrm{KCNH} 2$, but not $\mathrm{KCNH} 3$ in pancreatic tumour tissues compared with normal tissues $\mathrm{p}=0.011$ for KCNH1 and $\mathrm{p}=0.015$ for $\mathrm{KCNH} 2$ ). Overall, adenocarcinomas exhibited higher levels of all three $\mathrm{KCNH}$ than ductal carcinomas of the pancreas. KCNH members do not appear to have a clearly link to the staging of pancreatic cancer and only showed a weaker link to the survival of the patients. In our cohort database, we have identified that All three $\mathrm{KCNH}$ were found to have significantly correlated with a tumour repressor molecule, MTSS1/MIM (Metastasis Supressor-1/Missing In Metastasis) ( $\mathrm{r}=0.602,0.469$ and 0.508 for $\mathrm{KCNH} 1$, $\mathrm{KCNH} 2$ and $\mathrm{KCNH} 3$, respectively, $\mathrm{p}<0.0001$ by Spearman test). It was further demonstrated that KCNH1 and MTSS1/ MIM collectively is a risk factor for the death of the patients (HR 1.607, $\mathrm{p}=0.017)$ in predicting pancreatic cancer related survival (Mean survival 15.8 \pm 2.6 months versus $28.1 \pm 3.8$ months for patients with high and low aberration of KCNH1 and MTSS1, $\mathrm{p}=0.008$ ).

Conclusion KCNH family is aberrantly expressed in clinical pancreatic cancer and together with MTSS1/MIM offer a prognostic factor for the survival of the patients.

\section{PTU-68 THE EXPRESSION AND CLINICAL SIGNIFICANCE OF MLN64 IN HUMAN PANCREATIC CANCER}

${ }^{1}$ Laijian Sui ${ }^{*}$, ${ }^{1}$ Lin Ye, ${ }^{2}$ Chunyi Hao, ${ }^{1}$ Yuxin Cui, ${ }^{2}$ Ke Ji, ${ }^{2}$ Jiafu Ji, ${ }^{1}$ Wen G Jiang. ${ }^{1}$ Cardiff University School of Medicine, Cardiff, Wales, UK, Cardiff, UK; ${ }^{2}$ Peking University Cancer Hospital and Institute and Key Laboratory for Carcinogenesis, China

10.1136/gutjnl-2021-BSG.270 